\title{
Monitoring of Erosive Tooth Wear with Intraoral Scanners In vitro
}

\author{
Corinna Witecy $^{\mathrm{a}}$ Carolina Ganss $^{\mathrm{a}}$ Bernd Wöstmann ${ }^{\mathrm{b}}$ Moritz B. Schlenz ${ }^{\mathrm{b}}$ \\ Maximiliane A. Schlenz ${ }^{\text {b }}$ \\ a Department of Conservative and Preventive Dentistry, Justus Liebig University Giessen, Giessen, Germany; \\ ${ }^{b}$ Department of Prosthodontics, Justus Liebig University Giessen, Giessen, Germany
}

\section{Keywords}

Erosive tooth wear · Intraoral scanner · Profilometry ·

Monitoring

\begin{abstract}
Intraoral scanners (IOS) have been used to quantify tooth wear, but so far they have not been systematically validated for monitoring of tissue loss. The aim of this in vitro study was to investigate whether progression of tissue loss can be detected with an IOS and whether IOS values agree with those obtained with noncontacting profilometry (PRO) serving as a standard method. Model jaws were mounted in a phantom head positioned in a dental chair. Flattened areas were prepared on the non-load-bearing cusps of the first molars (model teeth; $n=16$ ) in order to fix flat enamel samples with an experimental area and a reference area. After baseline PRO and IOS, the experimental enamel area was stepwise etched with $35 \% \mathrm{H}_{3} \mathrm{PO}_{4}$ gel $(4 \times 30 \mathrm{~s}$ and $4 \times 15 \mathrm{~s})$. After each etching, PRO and IOS was performed and the vertical tissue loss between the reference and experimental areas was measured, each at the same 3 measurement points. Furthermore, cupped cusps were simulated by stepwise prepara-
\end{abstract}

tion of the load-bearing cusps of the model teeth with a spherical diamond bur, and the maximum vertical depth after each preparation step was measured only by IOS. Trios3 (3Shape, Denmark), Carestream CS3600 (Carestream, USA) and an optical profilometer (MicroProf, Fries, Germany) were used to measure the flat areas of the enamel samples, whereas only IOS were used to measure curved surfaces on the load-bearing cupped cusps of the model teeth. The IOS data were analyzed with an external software (GOM Inspect, Germany) and with the respective internal IOS software. PRO revealed a mean $( \pm S D)$ tissue loss of $17.1 \pm 4.7 \mu \mathrm{m}$ after 30 -s etching steps and $10.1 \pm 5.1 \mu \mathrm{m}$ after the 15 -s etching steps. IOS and software types were able to detect the progression of tissue loss after each etching step ( $p \leq 0.001$ each); BlandAltmann plots revealed good agreement with PRO regardless of the order of tissue loss, and no systematic difference was found. Increasing cupped lesion depths were detected by all IOS, with no significant differences between IOS and analysis methods. IOS were able to detect small amounts of tissue loss under simulated clinical conditions and seem to be a promising tool for monitoring even initial erosive tooth wear.

(c) 2021 The Author(s)

Published by S. Karger AG, Basel
C 2021 The Author(s)

Published by S. Karger AG, Basel

This article is licensed under the Creative Commons Attribution 4.0 International License (CC BY) (http://www.karger.com/Services OpenAccessLicense). Usage, derivative works and distribution are permitted provided that proper credit is given to the author and the original publisher.
Corinna Witecy

Department of Conservative and Preventive Dentistry

Justus Liebig University Giessen, Schlangenzahl 14

DE-35392 Giessen (Germany)

corinna.witecy@dentist.med.uni-giessen.de 


\section{Introduction}

Erosive tooth wear is an increasingly important oral health issue [Schlueter and Luka, 2018]. Therefore, sensitive methods for early detection, individual monitoring, and gathering of incidence data, as well as for clinical studies on the effects of therapeutic agents and behavior change strategies, are urgently required. An established method for recording erosive tooth wear consists of the use indices, which usually combine diagnostic criteria and scores for grading the severity of lesions. The literature provides a large number of such index systems, but none has gained general acceptance [Bardsley, 2008]. A more recent suggestion of a standardized, validated index and a simple tool for clinical practice is the Basic Erosive Wear Examination (BEWE) [Bartlett et al., 2008], which has been used clinically but also to monitor erosive wear on study casts and their 3-D images [Alaraudanjoki et al., 2017; Marro et al., 2018, 2020; Wohlrab et al., 2019].

However, all of these index systems are semiquantitative, making quantification and monitoring of early erosive tooth wear in the order of micrometers difficult. An attempt to overcome this problem consisted of using acid-resistant markers adhesively bonded to the eroded tooth surface serving as reference for profilometric measurements [Bartlett et al., 1997; Schlueter et al., 2005]. However, the method is time consuming, has problems with marker retention, and lacks patient compliance.

A more suitable method would be to capture 3-D images using scanners, which has already been suggested as the preferred method for measurement of tooth wear [DeLong, 2006]. To monitor wear in various contexts, the majority of studies have used 3-D datasets of study models [Wulfman et al., 2018; O’Toole et al., 2019a; Marro et al., 2020]. However, dimensional changes in the materials might affect the accuracy, especially at the micrometer level. Furthermore, this indirect measurement requires a complex laboratory set-up with a model scanner, inspection software, and expert skills.

Meanwhile, IOS have been developed from the restorative field to diagnostic instruments, as some manufactures have implemented an additional software application that allows chairside alignment of 2 datasets in the IOS software. Thus, IOS have been used for direct wear measurements in vitro [Meireles et al., 2016; Hartkamp et al., 2017b; Kumar et al., 2019] and in vivo [Hartkamp et al., 2017a].

So far, however, intraoral scanning has not been systematically validated for detection and monitoring of small amounts of tissue loss in the context of erosive tooth wear.
Therefore, it was the primary aim of the present study to investigate whether IOS can detect the progression of tissue loss after consecutive steps of short acid etching of prepared flat enamel samples. Two different IOS were used in a simulated clinical setting. The resulting 3-D data sets were analyzed with an external laboratory software as well as with the respective internal software using the measurement tools of the IOS. The standard method for comparison was profilometry (PRO).

A secondary aim was to examine whether the different IOS as well as the different types of software were suitable for monitoring of the progression of cupped lesions on cusps, typical of erosive tooth wear, which were simulated by manual preparation of model teeth.

\section{Materials and Methods}

\section{Preparation of Enamel Samples}

Human caries-free third molars extracted for therapeutic reasons were collected. After cleaning, the teeth were stored in saturated aqueous thymol solution (Sigma-Aldrich Chemie $\mathrm{GmbH}$, Steinheim, Germany) at $4^{\circ} \mathrm{C}$. From the smooth surfaces, approximately 1-mm-thick longitudinal slices were prepared, ground flat, polished (diamond grinding and polishing discs of 30, 15, and 3 $\mu \mathrm{m}$; Bühler GmbH, Düsseldorf, Germany), and cut, resulting in enamel samples of $4 \times 3 \mathrm{~mm}(n=16)$.

For a reproducible highly standardized test setup, 4 grooves (mesial, distal, oral, and buccal) were prepared with a diamond disc (ISO 806104317524220; Gebr. Brasseler GmbH \& Co. KG, Lemgo, Germany) on each enamel sample, serving as reference points (Fig. 1a).

Samples were checked for cracks and damage under a stereo microscope ( $\times 10$ magnification, Nikon SMZ-2T; Tokyo, Japan). The bottom sides of the enamel samples were etched with $35 \%$ $\mathrm{H}_{3} \mathrm{PO}_{4}$ gel for $30 \mathrm{~s}$ (iBond etch 35; Kulzer GmbH, Hanau, Germany), cleaned under running distilled water, and gently air dried.

\section{Fixing the Enamel Samples on the Model Teeth}

First, 4 first molar model teeth (ANA-4; frasaco $\mathrm{GmbH}$, Tettnang, Germany; FDI 16, 26, 36, and 46) were prepared. The non-load-bearing cusp of each model tooth was flattened with a diamond bur (ISO 806104140524880/018; Busch, Engelskirchen, Germany) and the teeth were mounted in the corresponding upper or lower model jaw (ANA-4; frasaco $\mathrm{GmbH}$ ), simulating a complete dentition. Then an enamel sample was luted with composite (Venus Diamond Flow; Heraeus Kulzer, Hanau, Germany) on the flattened area of each model tooth (Fig. 1a). While mounted with the uncured composite, profilometric traces were made on each enamel sample until it was assured that the enamel sample was positioned in parallel to the profilometer table and then fixed by light curing (120 s, Excelite-S white; Jovident GmbH, Duisburg, Germany). Four model jaw sets were created.

\section{Experimental Procedure}

At first, baseline profilometric measurements of the enamel samples were made. To simulate a real patient situation, the mod- 


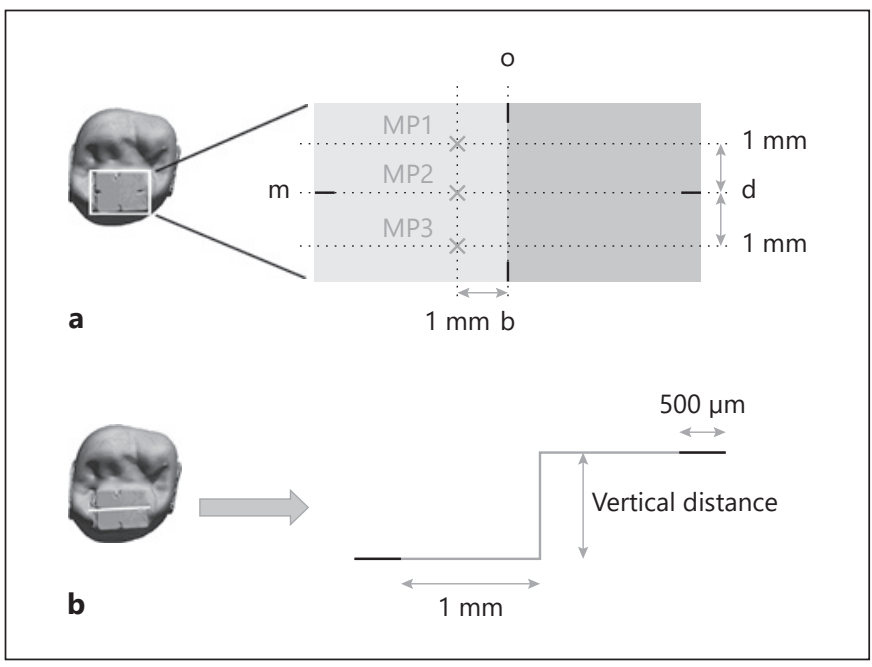

Fig. 1. a Schematic drawing of an enamel sample (occlusal perspective). Reference grooves were prepared on the oral (o), buccal (b), mesial (m), and distal (d) sides. The measuring areas were oriented in line with the $\mathrm{m} / \mathrm{d}$ grooves as well as $1 \mathrm{~mm}$ apart in both the oral and buccal directions. The 3 measuring points (MP1, MP2, and MP3) were located on the measurement areas $1 \mathrm{~mm}$ apart from the border between the experimental area (light grey) and the reference area (dark grey) of the enamel sample; b Schematic drawing of profilometric writing with the corresponding evaluation area (buccal-oral perspective). Two regression lines were created $1 \mathrm{~mm}$ apart from the border of the reference and the experimental area. Tissue loss $(\mu \mathrm{m})$ was defined as the vertical distance of the regression lines.

el jaws were mounted in a frasaco phantom head (P-6/5 HGB) equipped with a face mask (P-6 GMN). The phantom head was placed in a phantom torso (P-6-TSE) which was mounted in a dental chair for intraoral scanning. Two different IOS (TRI: Trios 3, version 1.18.2.10, 3Shape, Copenhagen, Denmark; CAR: Carestream CS3600, version 3.1.0, Carestream Dental LLC, Atlanta, GA, USA) were used. The scan area included the first molars with the mesial and distal adjacent teeth. The same scanning protocol was used for all scanning procedures, beginning with the occlusal and oral surface followed by the buccal surface [Müller et al., 2016]. Before scanning, TRI was calibrated using the respective calibration device based on the manufacturer's instructions. For CAR, no calibration was necessary.

After baseline PRO and intraoral scanning (T0), enamel loss was created. To protect the reference area, a transparent tape (tesafilm; Global Headquarters - tesa SE, Norderstedt, Germany) was applied under a surgical microscope ( $\times 5$ magnification,; Carl Zeiss Surgical GmbH, Oberkochen, Germany). Then the experimental area was etched with $35 \% \mathrm{H}_{3} \mathrm{PO}_{4}$ gel for $30 \mathrm{~s}$, cleaned under running distilled water for $30 \mathrm{~s}$, and gently air dried. Afterward, the tape was removed.

In addition, a cup was prepared on the mesial load-bearing cusps of each first molar model tooth using a spherical diamond bur (ISO 806104001524018; Busch) under constant water cooling. After that, the teeth were scanned as described above, the model jaws were removed from the phantom head, and PRO of the enamel samples was done. Subsequently, the enamel samples were etched and the cusps prepared again, and the model jaws were replaced in the phantom head. This procedure was repeated 4 times (T1 to T4). After T4, the enamel etching time was reduced to $15 \mathrm{~s}$ while all other procedures were kept constant and the procedure was repeated an additional 4 times (T5 to T8).

\section{Measurement of Enamel Loss}

Profilometric measurements were done with a noncontact optical device (MicroProf; Fries Research \& Technology GmbH, Bergisch-Gladbach, Germany; sensor HO, 200 pixels, 32 Hertz, vertical range of measurement: $300 \mu \mathrm{m}$, vertical resolution: $10 \mathrm{~nm}$, lateral resolution: $1-2 \mu \mathrm{m})$. Three traces at intervals of 1 and $3 \mathrm{~mm}$ in length were made and interpreted with special software (Mark III, Fries Research \& Technology $\mathrm{GmbH}$ ). On the traces, regression lines $0.5 \mathrm{~mm}$ in length and $1 \mathrm{~mm}$ apart from the border of the reference and the experimental area were constructed on both areas (Fig. 1b). After orientation on the $\mathrm{x}$ and $\mathrm{y}$ coordinates, tissue loss $(\mu \mathrm{m})$ was defined as the vertical distance of the regression lines. For a reproducible highly standardized test setup, all measurements of the flat enamel samples were taken at the same 3 measurement points (MP1, MP2, and MP3). The 3 measuring points were defined as intersections between constructed guides on each sample. The distance between the 3 measurement points, the outer borders, and the border between the experimental area (light grey) and the reference area (dark grey) of the enamel sample was $1 \mathrm{~mm}$ (Fig. 1a).

Datasets of the IOS (T0 to T8) from TRI and CAR were analyzed with an external 3-D measurement software (TRIe and CARe) as well as with the internal software using measuring tools of the IOS (TRIi and CARi).

For the former analysis with external software, data sets from IOS were exported in standard tessellation language to GOM Inspect 3-D software (version V8 SR1; GOM GmbH, Braunschweig, Deutschland) and reduced to the area of interest (first molar). Then, scans were analyzed by superimposition of the baseline scan (T0) with each of the consecutive datasets (T1 to T8) using best-fit alignment (iterative closest point technique). Only 2 datasets were superimposed at one time (e.g., T0 and T1, T0 and T2, T0 and T3, $\ldots, \mathrm{T} 0$ and T8). Construction tools such as planes in the 3-D software were used to define the exact measurement points (MP1, MP2, and MP3) on the baseline dataset (T0) to ensure a standardized measurement. The maximum vertical loss $(\mu \mathrm{m})$ for the etched area of enamel was measured at the measuring points (MP1-3) as described in Figure 1.

The internal software of both IOS was used for superimposing the follow-up scans with the baseline scans as described for the external analysis, and the respective measuring tools were used to determine the enamel loss $(\mu \mathrm{m})$ at the same measurement points (MP1-3) as described above.

\section{Measurement of Cusp Loss}

The maximum depth $(\mu \mathrm{m})$ of the cupped lesion of the cusps (cusp loss) was measured on the 3-D datasets obtained from the 2 IOS either with GOM or with the respective internal software and measuring tools of the IOS. PRO was not performed.

\section{Reproducibility}

Before starting the experimental procedure, the reproducibility was determined. One additional enamel sample was prepared, 
Fig. 2. Representative illustration of the superimposed data sets from a right upper first molar showing the difference from T0 to T4 (top) and from T0 to T8 (bottom) as obtained from the 2 scanner types (TRI and CAR) and from the external (e) and internal (i) software. Differences between the experimental (left) and the reference (right) areas of the enamel sample as well as of the cup on the load bearing cusp can be clearly seen.

Table 1. Reproducibility of results from 10 repeated measurements of the same sample obtained from the 2 IOS types and from the external and internal software

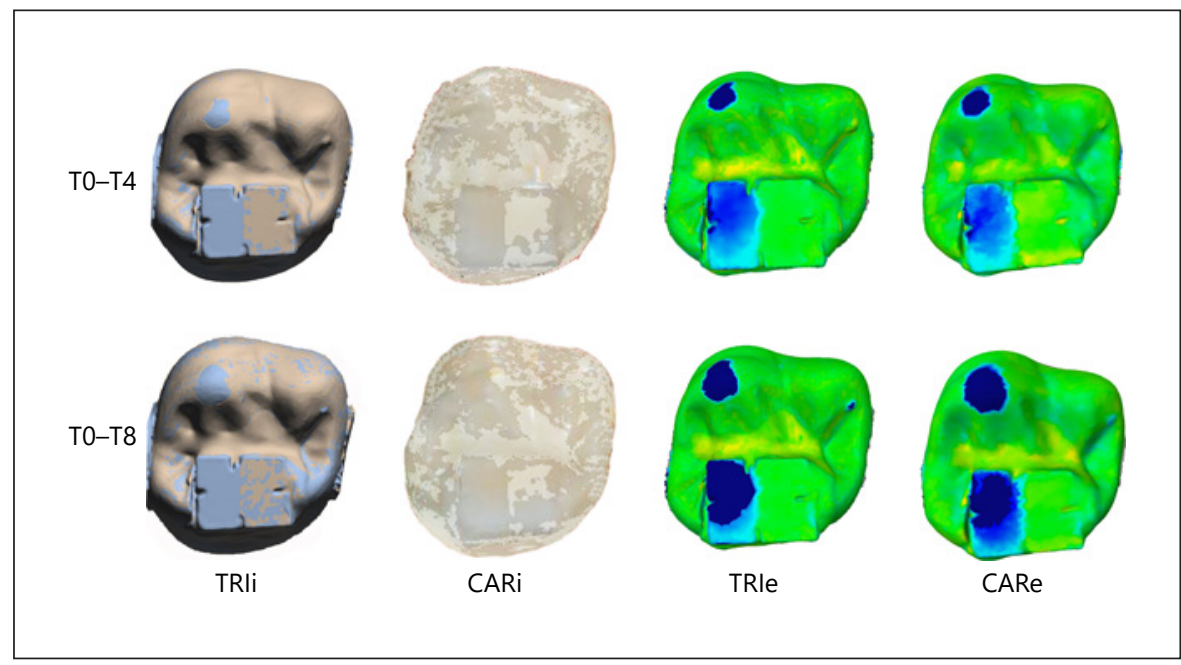

\begin{tabular}{llllll}
\hline & PRO & TRIe & CARe & TRIi & CARi \\
\hline Mean \pm SD, $\mu \mathrm{m}$ & $34.2 \pm 0.3$ & $23.9 \pm 2.4$ & $25.8 \pm 3.0$ & $23.0 \pm 3.7$ & $22.3 \pm 3.7$ \\
$99 \%$ CI & $33.9-34.5$ & $21.4-26.3$ & $22.7-28.9$ & $19.2-26.8$ & $18.8-25.7$ \\
\hline
\end{tabular}

mounted on a model tooth in a complete jaw model as described before, and, after baseline measurements, etched with phosphoric acid for $60 \mathrm{~s}$. To simulate a comparable experimental procedure, measurements were taken beginning with PRO, followed by mounting of the model in a phantom head and taking of intraoral scans with Trios 3 and CS3600. After the last measurement, the models were removed from the phantom head and the whole procedure was repeated 10 times with all 3 devices (PRO, TRI, and CAR). Loss values as well as 99\% CI are given in Table 1.

\section{Statistics}

Statistics were done with IBM SPSS Statistics version 25 (IBM Germany GmbH, Ehningen, Germany). All data were checked for deviations from a Gaussian distribution (Kolmogrorov-Smirnov test). As there were no significant deviations, parametric procedures were used for all comparisons. Values are given as means \pm SD. $p<0.05$ was considered statistically significant.

\section{Enamel Loss}

The loss of a sample was expressed as the mean of 3 measurements ( $\mu \mathrm{m}$; MP1-3). Within-method comparisons of cumulative loss values from T1 to T8 were done with $t$ tests for dependent samples. In addition to the cumulative loss values, their differences from the respective previous value were calculated to describe the loss occurring from each etching step. One-sample $t$ tests and 99\% CI were used to investigate whether these differences were different from zero. Bland-Altman plots [Bland and Altman, 1999; Giavarina, 2015] were used to analyze the agreement of enamel loss values after each etching step obtained from IOS (TRIe, CARe, TRIi, and CARi), with values from PRO as the reference. A regression analysis was performed to evaluate whether the order of val- ues had an effect on agreement (proportional difference), and onesample $t$ tests of the mean differences of values obtained from the 4 methods with values from PRO were conducted to evaluate whether there was a systematic difference.

\section{Cusp Loss}

Cusp loss was determined as the maximum depth of the cupped lesion $(\mu \mathrm{m})$. Within-method comparisons of cumulative loss values from T1 to T8 were done with $t$ tests for dependent samples. In addition, as described above, differences from the respective previous value were calculated to describe the loss occurring from each preparation step. The cusp loss occurring after each preparation step obtained from the 2 IOS and software types (TRIe, CARe, TRIi, and CARi) was compared by $t$ tests for dependent samples (between-method comparisons).

\section{Results}

\section{Enamel Loss}

Figure 2 shows an example of the superimposed data sets from the 2 IOS as well as from the external and internal analyses.

The data for the cumulative enamel loss from T1 to T8 obtained from all measuring methods is shown in Fig. 3. $\mathrm{PRO}$ revealed a significant increase in enamel loss after each etching for both the 30-s and the 15-s etching times ( $p \leq 0.001$ each). Similar results were found with intraoral 


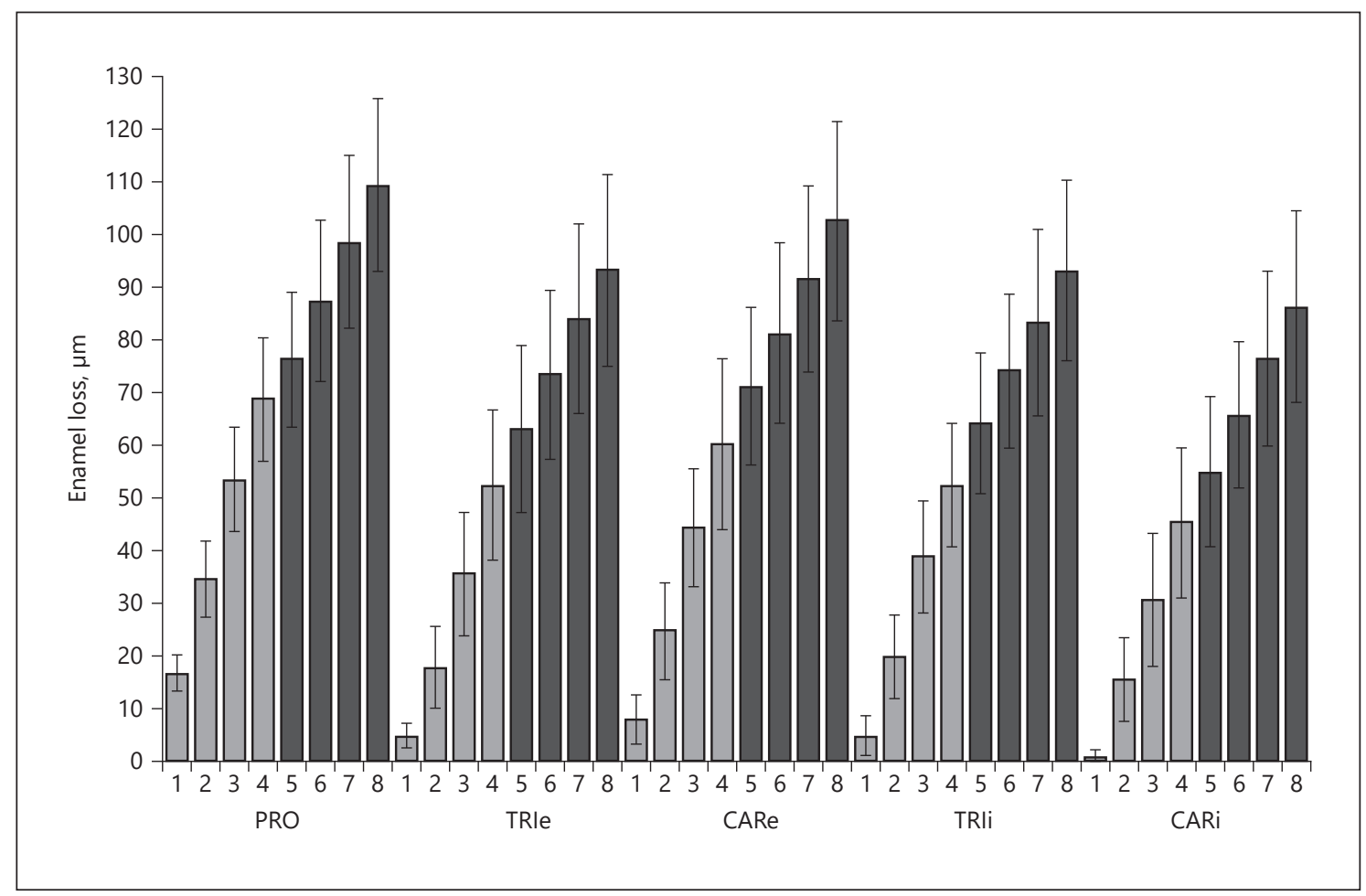

Fig. 3. Cumulative enamel loss (mean \pm SD) after each of the 8 consecutive etching steps (light grey: 30 -s etching time; dark grey: 15 -s etching time) as obtained from the 2 scanner types (TRI and CAR) and from the external (e) and internal (i) software.

Table 2. Mean differences and $99 \%$ CI of loss values occurring after each etching step

\begin{tabular}{lllllllrr}
\hline & T1-T0 & T2-T1 & T3-T2 & T4-T3 & \multicolumn{1}{l}{ T5-T4 } & T6-T5 & T7-T6 & T8-T7 \\
\hline PRO & $16.6(14.2-19.1)$ & $17.8(14.4-21.1)$ & $18.8(15.1-22.5)$ & $15.1(11.1-19.1)$ & $7.6(4.3-10.8)$ & $11.0(7.4-14.6)$ & $11.1(6.7-15.6)$ & $10.7(7.2-14.2)$ \\
TRIe & $4.8(3.2-6.5)$ & $12.8(6.8-18.9)$ & $17.7(14.0-21.5)$ & $16.7(12.9-20.5)$ & $10.5(7.3-13.6)$ & $10.4(8.2-12.6)$ & $10.6(7.9-13.3)$ & $9.2(6.7-11.7)$ \\
CARe & $7.8(4.3-11.3)$ & $16.9(11.6-22.1)$ & $19.6(15.5-23.6)$ & $15.7(10.5-20.9)$ & $10.8(7.5-14.1)$ & $10.0(7.0-13.0)$ & $10.3(7.4-13.2)$ & $10.9(8.7-13.1)$ \\
TRIi & $4.8(2.0-7.6)$ & $14.8(8.5-21.1)$ & $19.0(13.9-24.0)$ & $13.5(8.8-18.3)$ & $11.7(7.1-16.2)$ & $10.0(2.6-17.4)$ & $9.0(4.1-13.8)$ & $9.8(5.7-14.0)$ \\
CARi & $0.6(-0.5-1.6)$ & $14.7(9.1-20.4)$ & $15.1(9.3-20.9)$ & $14.6(9.3-19.8)$ & $9.7(7.3-12.0)$ & $10.6(6.9-14.3)$ & $10.6(5.2-15.9)$ & $10.0(6.6-13.3)$ \\
\hline
\end{tabular}

T1 to T4: 30-s etching time. T5 to T8: 15-s etching time.

scanning regardless of the scanner type or the mode of analysis (within each method all cumulative increases $p \leq$ $0.001)$. Further, the differences from the previous etching step were all significantly different from zero $(p \leq 0.001$ for all differences except CARi at T1 n.s.; Table 2).

The Bland-Altman analysis (Fig. 4) revealed that for all comparisons except TRIi the slope of the regression line was not significantly different from zero, indicating that the order of loss values did not influence the agreement of the scanner results with the results from PRO. For TRIi, there was a small but significant $(p \leq 0.01)$ tenden- cy for higher values at a smaller scale and lower values at a greater scale. For all comparisons, the mean differences were not significantly different from zero, indicating that neither method had a systematic difference compared to PRO; thus the variability of the differences was only linked to the repeatability imprecision of each of the methods of comparison. Due to this, the limits of agreement indicate that, in a single case, an IOSe measurement can be approximately $\pm 10 \mu \mathrm{m}$ of the PRO measurement and an IOSi measurement can be $\pm 15 \mu \mathrm{m}$ of the PRO measurement. 


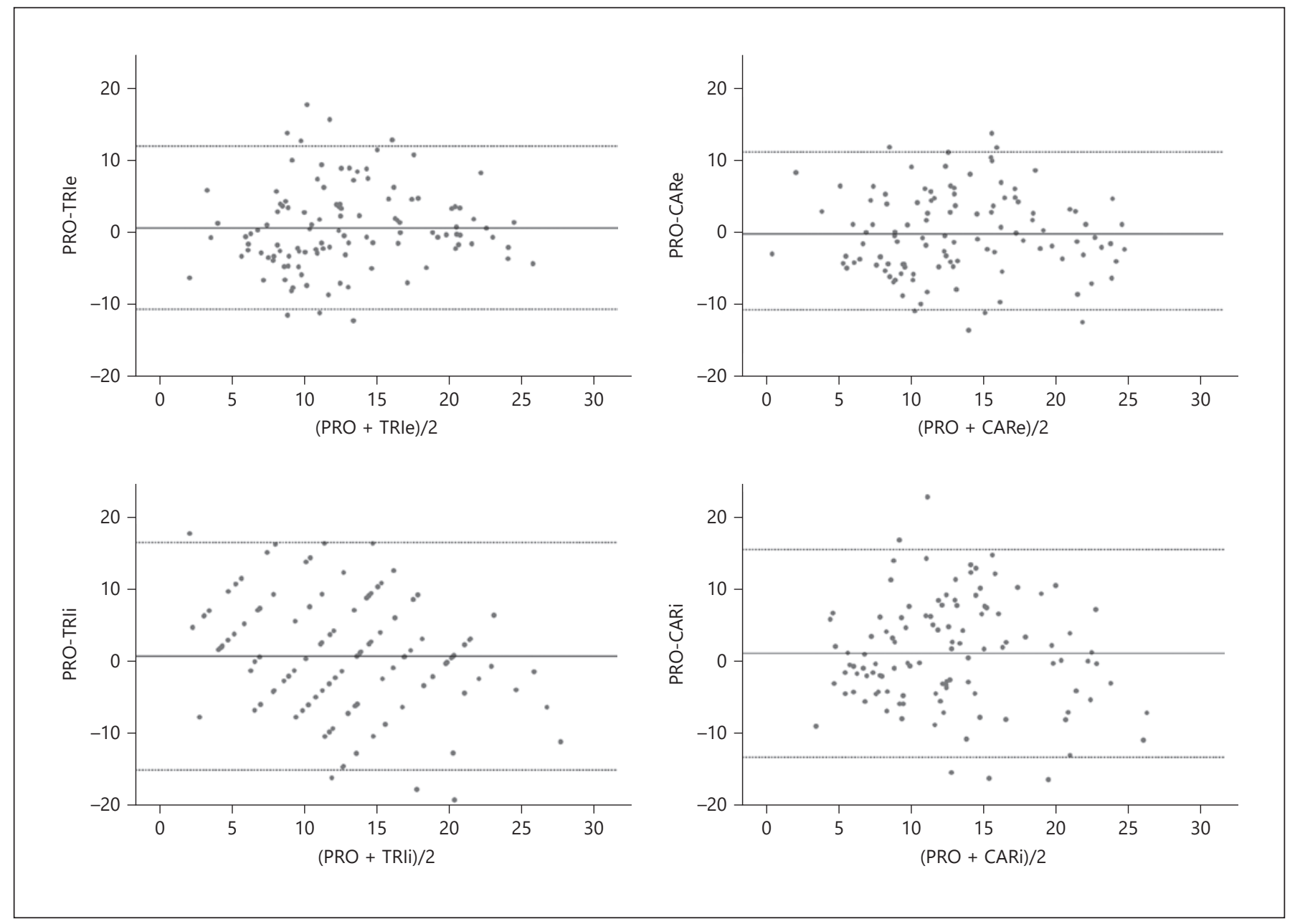

Fig. 4. Bland-Altman plots evaluating the agreement between the loss values $(\mu \mathrm{m})$ obtained from the 2 scanner types (TRI and CAR) and from the external (e) and internal (i) software with PRO. The solid line indicates the mean difference of the methods of comparison; the broken lines indicate the $95 \%$ limits of agreement (mean \pm $1.96 \times \mathrm{SD}$ ). Please note that the data output software TRIi on the micrometer scale gives no output on decimal places.

\section{Cusp Loss}

All IOS were able to detect cusp loss (Fig. 5) from manual bur preparation as cumulative loss values increased significantly from time point to time point (within each method, all differences $p \leq 0.0001$ ). The overall loss per preparation step was $55.0 \pm 32.8,55.3 \pm 35.2,56.4 \pm 36.4$, and $55.9 \pm 36.9 \mu \mathrm{m}$ for TRIe, CARe, TRIi, and CARi, respectively. From time point to time point (T2-T1, T3T2, ... T8-T7), both IOS and methods of analysis revealed similar loss values, with no significant differences between them.

\section{Discussion}

As a primary aim, the present study investigated the potential of IOS for detecting progression of small amounts of tissue loss in comparison to PRO as a reference [Schlueter et al., 2011]. To simulate a setting close to the clinical situation, model teeth were mounted in a phantom head positioned in a dental chair, where all IOS procedures were performed. Flat enamel samples were used as they fulfill the requirements for profilometric measurements in the order of a few micrometers; in addition, enamel allows for creation of tissue loss through acid etching at any desired dimension and in a suitable way. The profilometric procedure we used here 


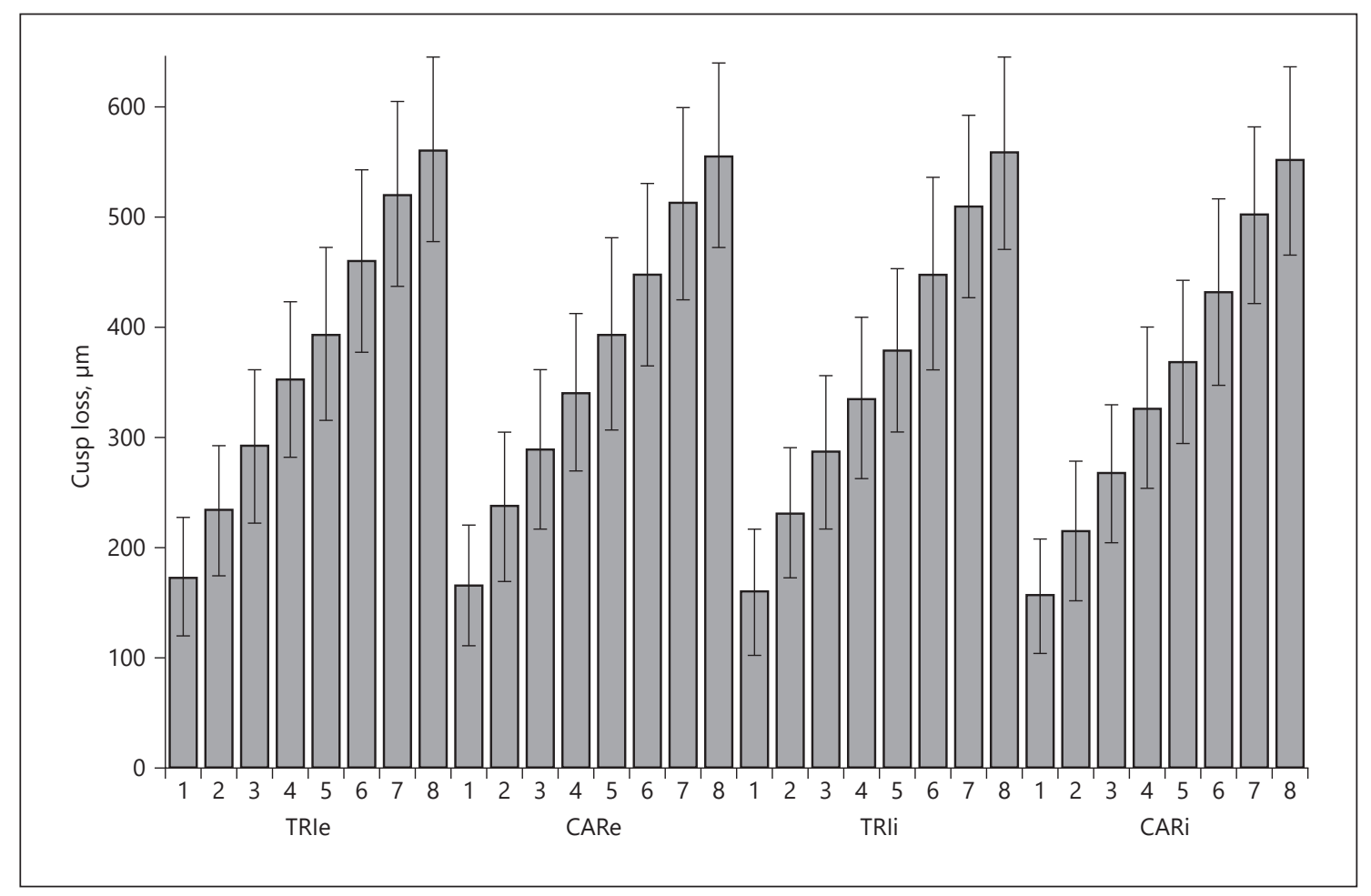

Fig. 5. Cumulative cusp loss (mean $\pm \mathrm{SD}$ ) after each of the 8 consecutive preparation steps as obtained from the 2 scanner types (TRI and CAR) and from the external (e) and internal (i) software.

has been validated [Ganss et al., 2005] and it has been used in numerous studies of our group, showing that it is able to measure tissue loss in various dimensions in a reliable way. The magnitude of tissue loss was chosen in view of clinical needs for monitoring, i.e., reasonable observation periods, but also in view of needs for discriminating physiological from pathological wear. So far, there is no age-related threshold for pathological wear rates; therefore, the different resistance of enamel and dentine against chemical and physical impacts was considered. On exposed dentine, antierosion agents are limited in efficacy [Magalhaes et al., 2011] and physical forces lead to higher wear rates in dentine than in enamel [Dzakovich and Oslak, 2017]. Therefore, one pillar in tooth wear prevention should be preservation of the enamel covering of the crown. On occlusal surfaces of premolars and molars, the enamel has a thickness in the order of 1-2 mm [Kono et al., 2002]; thus, from a young age, an annual wear rate of no more than about $20 \mu \mathrm{m}$ may be considered acceptable. This order of wear was also described in a convenience sample of young adults with complete dentition and normal occlusion [Lambrechts et al., 1989].

Monitoring Erosive Tooth Wear with IOS
The obtained 3-D datasets were further processed with different software. For comparison of 3-D datasets, the alignment procedure is important to obtain a rigid transformation that best adapts 2 existing models [Meireles et al., 2016]. The superimposition, however, is not trivial and it is prone to error [Besl, 1992], and the choice of alignment method has a significant influence on the measurement outcome. In contrast to reference alignment, best-fit alignment minimizes the mesh distance error between 2 data sets and may underestimate the order of a difference at the area of interest [O'Toole et al., 2019b]. However, there is no reliable reference structure in the oral cavity. Because the internal IOS software uses best-fit alignment, the same alignment procedure was used for the external analysis with 3-D laboratory software for standardized testing conditions. The authors are aware of a minimized mesh distance due to best-fit alignment, but in this study a progression of flat and cupped lesions was shown regardless of the software. From a clinical point of view, this is an important gain of information for daily practice.

As expected, the enamel loss increased with time and the order of it varied according to the varying susceptibility of the human material coming from different donors 
[Uhlen et al., 2016]. Thus, a clinically relevant order of variation of the measured values was obtained, which provided a good source for validation. PRO was able to detect this tissue loss after each 30 -s and 15 -s etching step (Fig. 3). Even though a linear increase and reproducible results were also found for IOS, absolute loss values between PRO and IOS differed.

The authors are aware of the problem that measurement of tissue loss in the order of micrometers might represent in the area of measurement uncertainty of IOS. Manufacturers do not quote the exact number of measurement points for IOS datasets. However, some studies have focused on that topic and investigated the resolution of IOS used in this study. Chiu et al. [2020] described a scanning resolution in the region of $33 \mu \mathrm{m}$ for Trios 3 regarding discrepancy on the finish line. A study of Medina-Sotomayor et al. [2018] analyzed the relationship between resolution and accuracy (trueness/precision) of different IOS. They described a resolution in the region of $40 \mu \mathrm{m}$ for Trios 3 but could not find a relationship between resolution and accuracy.

Monitoring, however, means detecting a difference in tissue loss from different observations; thus the focus of this paper was not to describe absolute values but rather to show that detection of consecutive tissue loss in a clinically meaningful dimension is possible with IOS. This has a high clinical relevance because treatment options depend on progression or stagnation of substance loss.

External analysis with an elaborated 3-D laboratory software tool like GOM is time consuming and requires operator skills. In view of the need for routine applications, we additionally examined the internal measuring software of the IOS, which is easy to use and already clinically applicable for analysis of fit regarding single crowns [Schlenz et al., 2020]. In view of clinical needs, it is an encouraging finding that the internal software was also able to detect tissue loss even after the 15-s etching steps with good agreement with the profilometer results. The limitation was that the output of the current internal software of TRI was on a millimeter scale with only 2 decimal places. This means that on the micrometer scale tissue loss was given in decimal steps, which explains the proportional difference to PRO. IOS software should be improved to report measurements on a micrometer scale.

A limitation of the present study is that the analysis was restricted to the vertical height loss on sectional planes. Tooth wear varies distinctly with respect to the shape of the lesions; thus, tissue loss can be reasonably described as changes in depth, volume, or affected area.
Accordingly, parameters like volume change, mean and maximum surface height loss, average profile height loss, or percentage of the surface area affected by wear have been used for quantification. In addition to the options of IOS measurement tools, a current easy-to-use free software offering more measuring parameters could be useful for a more comprehensive description of tooth wear (www.leadsdigitaldentistry.com/WearCompare) [O'Toole et al., 2019b]. However, as no particular parameter has gained general acceptance, a recent systematic review on the methodology of wear measurement concluded that there is need for standardization [Wulfman et al., 2018]. Therefore, an important issue for future research is validation of various parameters or combinations of them for monitoring of erosive tooth wear.

So far, results of measurement of flat enamel samples have been regarded. In the clinical situation, however, such defined flat areas usually do not occur, with the exception, perhaps, of facets from attrition. In addition to the measurements on gradually etched enamel, scanners were therefore also applied to a clinically relevant defect form as a secondary aim. For this purpose, cupped lesions of cusps were simulated by manual bur preparation and the resulting substance loss was evaluated with the 2 scanners and different types of software each. The measurement problem here was not the determination of measurement limits but the question of whether the scanners would measure the progressive loss of cupped lesions in a comparable way. The application of PRO would not have yielded any further insight here. Manual preparation produced substance loss values in the order of about $50 \mu \mathrm{m}$ at each time point (Fig. 5), which is clearly within the measuring capacity of the scanners as determined with the flat samples. The relatively wide range of values obtained is due to the manual preparation, which in itself creates a greater variability of values than etching. All measurement and analysis procedures were able to show the increasing depth of the lesion with time, and there were no significant differences between them. An encouraging result is that the internal software of both scanners again produced results comparable to those of the external evaluation. Thus, the scanners could potentially be an easy-to-use method to monitor cupped lesions in dental practice.

Clearly, a laboratory set-up can only simulate clinical conditions partially. This is a limitation as natural teeth sometimes incur wear over the entire crown, lacking stable reference areas, or are prone to adaptive movements for instance elongation. Furthermore, clinical conditions (e.g., saliva or patient movement) might influence the 
data acquisition of IOS. However, a recent study investigating pre- and postorthodontic treatment casts demonstrated that IOS was able to confirm clinically visible wear progression [Marro et al., 2020], indicating the potential of IOS for wear monitoring. Further research, however, is required to refine methods of alignment and analysis and to investigate whether the promising measurement potential of the scanners shown here can also be realized in clinical practice.

\section{Conclusion}

In a simulated clinical setting, IOS were able to detect progression of tissue loss on flat enamel samples even after 15-s etching steps both when the datasets were analyzed with an external software (GOM) and with the respective internal software tools. Bland-Altman analysis revealed good agreement with tissue loss values obtained from PRO, which served as the reference method. Monitoring of the progression of tissue loss from cupped lesions was also possible with both IOS and software types. IOS seem to be a promising tool to monitor erosive tooth wear even at small wear rates.

\section{Statement of Ethics}

Teeth were extracted with the patients' consent. The use of human extracted teeth was approved by the Ethics Committee of the Justus Liebig University of Giessen (ref. No. 143/09).

\section{Conflict of Interest Statement}

The authors have no conflict of interests to declare.

\section{Funding Sources}

The study was funded by the Justus Liebig University of Giessen.

\section{Author Contributions}

C.W. and C.G. share first authorship. C.W. designed and performed the experiment, acquired profilometric and IOS data, and wrote this paper. C.G. designed the experiment, performed statistical analyses, interpreted the data, and wrote this paper. B.W. allocated the resources and revised this paper. M.B.S. performed the experiments, acquired IOS data, and revised this paper. M.A.S. designed the experiment, monitored the data acquisition, and wrote this paper. All of the authors approved the final version of this paper and are accountable for all aspects of this work.

\section{References}

Alaraudanjoki V, Saarela H, Pesonen R, Laitala ML, Kiviahde H, Tjäderhane L, et al. Is a Basic Erosive Wear Examination (BEWE) reliable for recording erosive tooth wear on $3 \mathrm{D}$ models? J Dent. 2017 Apr;59:26-32.

Bardsley PF. The evolution of tooth wear indices. Clin Oral Investig. 2008 Mar; 12(Suppl 1):S15-9.

Bartlett DW, Blunt L, Smith BG. Measurement of tooth wear in patients with palatal erosion. $\mathrm{Br}$ Dent J. 1997 Mar;182(5):179-84.

Bartlett D, Ganss C, Lussi A. Basic Erosive Wear Examination (BEWE): a new scoring system for scientific and clinical needs. Clin Oral Investig. 2008 Mar;12(Suppl 1):S65-8.

Besl PJ, McKay ND. A method for registration of 3-D shapes. IEEE Trans Pattern Anal Mach Intell. 1992;14(2):239-56.

Bland JM, Altman DG. Measuring agreement in method comparison studies. Stat Methods Med Res. 1999 Jun;8(2):135-60.

Chiu A, Chen YW, Hayashi J, Sadr A. Accuracy of CAD/CAM Digital Impressions with Different Intraoral Scanner Parameters. Sensors (Basel). 2020 Feb;20(4):20.

DeLong R. Intra-oral restorative materials wear: rethinking the current approaches: how to measure wear. Dent Mater. 2006 Aug;22(8): 702-11.
Dzakovich JJ, Oslak RR. In vitro effects of acid challenge on incisal/occlusal cupping/cratering. J Prosthet Dent. 2017 Jan;117(1):124-31.

Ganss C, Lussi A, Klimek J. Comparison of calcium/phosphorus analysis, longitudinal microradiography and profilometry for the quantitative assessment of erosive demineralisation. Caries Res. 2005 May-Jun;39(3): 178-84.

Giavarina D. Understanding Bland Altman analysis. Biochem Med (Zagreb). 2015 Jun;25(2): 141-51.

Hartkamp O, Lohbauer U, Reich S. Antagonist wear by polished zirconia crowns. Int J Comput Dent. 2017a;20(3):263-74.

Hartkamp O, Peters F, Bothung H, Lohbauer U, Reich S. Optical profilometry versus intraoral (handheld) scanning. Int J Comput Dent. 2017b;20(2):165-76.

Kono RT, Suwa G, Tanijiri T. A three-dimensional analysis of enamel distribution patterns in human permanent first molars. Arch Oral Biol. 2002 Dec;47(12):867-75.

Kumar S, Keeling A, Osnes C, Bartlett D, O’Toole $\mathrm{S}$. The sensitivity of digital intraoral scanners at measuring early erosive wear. J Dent. 2019 Feb;81:39-42.
Lambrechts P, Braem M, Vuylsteke-Wauters M, Vanherle G. Quantitative in vivo wear of human enamel. J Dent Res. 1989 Dec;68(12): 1752-4.

Magalhães AC, Wiegand A, Rios D, Buzalaf MA, Lussi A. Fluoride in dental erosion. Monogr Oral Sci. 2011;22:158-70.

Marro F, De Lat L, Martens L, Jacquet W, Bottenberg P. Monitoring the progression of erosive tooth wear (ETW) using BEWE index in casts and their 3D images: A retrospective longitudinal study. J Dent. 2018 Jun;73:70-5.

Marro F, Jacquet W, Martens L, Keeling A, Bartlett D, O'Toole S. Quantifying increased rates of erosive tooth wear progression in the early permanent dentition. J Dent. 2020 Feb; 93:103282.

Medina-Sotomayor P, Pascual-Moscardó A, Camps I. Relationship between resolution and accuracy of four intraoral scanners in complete-arch impressions. J Clin Exp Dent. 2018 Apr;10(4):e361-6.

Meireles AB, Vieira AW, Corpas L, Vandenberghe B, Bastos FS, Lambrechts P, et al. Dental wear estimation using a digital intra-oral optical scanner and an automated 3D computer vision method. Comput Methods Biomech Biomed Engin. 2016;19(5):507-14. 
Müller P, Ender A, Joda T, Katsoulis J. Impact of digital intraoral scan strategies on the impression accuracy using the TRIOS Pod scanner. Quintessence Int. 2016 Apr;47(4):343-9.

O’Toole S, Osnes C, Bartlett D, Keeling A. Investigation into the validity of WearCompare, a purpose-built software to quantify erosive tooth wear progression. Dent Mater. 2019a Oct;35(10):1408-14.

O’Toole S, Osnes C, Bartlett D, Keeling A. Investigation into the accuracy and measurement methods of sequential 3D dental scan alignment. Dent Mater. 2019b Mar;35(3):495-500.
Schlenz MA, Vogler J, Schmidt A, Rehmann P, Wöstmann B. New Intraoral Scanner-Based Chairside Measurement Method to Investigate the Internal Fit of Crowns: A Clinical Trial. Int J Environ Res Public Health. 2020 Mar;17(7):17

Schlueter N, Ganss C, De Sanctis S, Klimek J. Evaluation of a profilometrical method for monitoring erosive tooth wear. Eur J Oral Sci. 2005 Dec;113(6):505-11.

Schlueter N, Hara A, Shellis RP, Ganss C. Methods for the measurement and characterization of erosion in enamel and dentine. Caries Res. 2011;45 Suppl 1:13-23.

Schlueter N, Luka B. Erosive tooth wear - a review on global prevalence and on its prevalence in risk groups. Br Dent J. 2018 Mar: 224(5):364-70.
Uhlen MM, Mulic A, Holme B, Tveit AB, Stenhagen KR. The Susceptibility to Dental Erosion Differs among Individuals. Caries Res. 2016; 50(2):117-23.

Wohlrab T, Flechtenmacher S, Krisam J, Saure D, Wolff D, Frese C. Diagnostic value of the Basic Erosive Wear Examination for the assessment of dental erosion on patients, dental photographs, and dental casts. Oper Dent. 2019 Nov/Dec;44(6):E279-88.

Wulfman C, Koenig V, Mainjot AK. Wear measurement of dental tissues and materials in clinical studies: A systematic review. Dent Mater. 2018 Jun;34(6):825-50. 